\title{
Spatial S-R compatibility effects in an intentional imitation task
}

\author{
CECILIA HEYES and ELIZABETH RAY \\ University College London, London, England
}

\begin{abstract}
The active intermodal mapping hypothesis suggests that intentional imitation is mediated by a highly efficient, special-purpose mechanism of actor-centered movement encoding. In the present study, using methods from stimulus-response (S-R) compatibility research, we found no evidence to support this hypothesis. In two experiments, the performance of adult participants instructed to imitate actorcentered spatial properties of head, arm, and leg movements was affected by task-irrelevant, egocentric spatial cues. In Experiment 1, participants imitated using the same side of their bodies as did the model, and performance was less accurate when egocentric stimulus location was response incompatible than when it was response compatible. This effect was reversed in Experiment 2 when participants imitated using the opposite side of their bodies. These findings, in line with general process theories of imitation, imply that intentional imitation is mediated by the same processes that mediate responding to inanimate stimuli on the basis of arbitrary $\mathrm{S}-\mathrm{R}$ mappings.
\end{abstract}

Imitation has long been of interest to developmental and comparative psychologists, but it has recently also become a focus of inquiry in experimental psychology, cognitive neuroscience, and robotics (e.g., Dautenhahn \& Nehaniv, 2002; Prinz, 2002). A significant challenge for research in this area is to explain imitation of perceptually opaque actions, such as facial expressions and gross body movements, which give rise to disparate sensory input when observed and executed. Theories addressing this "correspondence problem" suggest either that the processes mediating stimulus-response (S-R) translation for imitation are "special," dedicated and innate (Meltzoff \& Moore, 1997), or that they are the same general processes that mediate responding to inanimate stimuli on the basis of arbitrary S-R mappings (Heyes, 2001; Prinz, 2002).

A prominent special-process theory, the active intermodal mapping (AIM) hypothesis, was originally formulated to explain facial gesture imitation in neonates, but has recently been applied to adults' imitation of appendage movements (e.g., Meltzoff \& Decety, 2003). It suggests that $\mathrm{S}-\mathrm{R}$ translation for imitation is mediated by a dedicated "supramodal representation system" which encodes sensory input from both observed and executed body movements as "organ relations" such as "tongue-betweenlips." The AIM hypothesis specifies that "organ relations are the lingua franca by which acts of self and other can be

This research was supported by the Economic and Social Research Council's Economic Learning and Social Evolution (ELSE) research center at University College London. We are grateful to Marie Shaftoe for assistance in data scoring, John McCarthy for software advice, and Martin Eimer, Bernhard Hommel, Wolfgang Prinz, and an anonymous reviewer for comments on a earlier draft of the article. Correspondence should be addressed to C. Heyes, Department of Psychology, University College London, Gower Street, London WC1E 6BT, UK (e-mail: c.heyes@ucl.ac.uk). commonly coded" (Meltzoff \& Moore, 1997). In other words, supramodal representations of organ relations are actor centered, rather than egocentric, in that they encode movement properties that are present both when the movement is observed and when it is executed, and therefore do not depend on the observer's point of view. AIM proposes that supramodal representation is specific to body movements, and that dynamic, inanimate stimuli are not encoded as organ relations. ${ }^{1}$ It also postulates that the basic capacity for supramodal representation is innate, and that it develops swiftly through experience so that even infants can "directly and fluidly imitate a novel act ... even though they have never practiced this particular movement pattern before" (Meltzoff \& Moore, 1997).

There are two reasons why it is difficult to derive distinctive empirical predictions from the AIM hypothesis that imitation is mediated by a special-purpose, supramodal representation system. First, the hypothesis does not specify a code in which organ relations are represented. It makes a claim about what is represented - organ relations - but, aside from denying that the code is modality specific, it does not indicate how this content is encoded. Second, the AIM hypothesis does not comment on how, if at all, the supramodal system interacts with other, more general processes of S-R translation. However, it is usually assumed that special-purpose mechanisms, such as those mediating vision and native language comprehension, are relatively encapsulated and therefore invulnerable to interference from more general processes (Fodor, 1983). Therefore, if imitation is mediated by a specialpurpose mechanism, and one that develops so fast that even infants are sophisticated imitators, one might expect adult imitative performance to be invulnerable to some of the sources of interference apparent in other, nonimitative S-R translation tasks. 
In the present study, we used an S-R compatibility task to examine this possibility. In a typical task of this kind, participants are instructed to press a left key when they see a red light and a right key when they see a green light, and are found to respond less efficiently (slower and/or less accurately) to stimuli presented contralateral to the correct response than to stimuli presented on the ipsilateral side (Lu \& Proctor, 1994; Wallace, 1971). Effects like this indicate that, in tasks involving inanimate stimuli and arbitrary S-R mappings, task-irrelevant egocentric spatial properties of the stimulus - the location of the stimulus relative to the participant - interfere with performance. If, as AIM suggests, imitation is mediated by a special-purpose process generating actor-centered representations of body movements, S-R compatibility effects of this kind might not be expected to occur when participants are instructed to imitate lateralized body movements.

Support for the hypothesis that imitative performance is invulnerable to S-R compatibility effects comes from two sources. Wapner and Cirillo (1968) showed that when children are told, "Do just like I do" by a model who is facing them and performing a lateralized body movement (e.g., raise right arm), 8-year-olds typically make an egocentrically compatible response (raise left arm), but the frequency of actor-centered responses (raise right arm) increases steadily with age until, at 18 years, $80 \%-85 \%$ of responses are actor centered. It would be surprising if adults freely choose a markedly less efficient response mode, and therefore it is plausible that this developmental trend reflects increasing invulnerability of actor-centered coding of body movements to interference from egocentric codes.

More direct evidence of invulnerability comes from a study in which adult participants were instructed to imitate, in an actor-centered fashion, a complex sequence of bimanually coordinated movements to tie knots in a rope (Sambrook, 1998). When the model was facing the observer, and therefore actor-centered and egocentric spatial codes were incompatible (e.g., movements of the model's right hand appeared on the observer's left), imitative performance was just as efficient as when the observer looked over the model's shoulder, and therefore actor-centered and egocentric spatial codes were compatible.

In contrast, other studies suggest that, like performance in nonimitative tasks, imitative performance is subject to S-R compatibility effects. For example, in a simple RT task in which the response consisted of lifting a finger, it was initiated sooner when the go signal was a lifting finger than when it was a tapping finger (Brass, Bekkering, \& Prinz, 2001). Similarly, participants instructed to respond to the color of a hand stimulus by opening or closing their own hands responded faster when the observed movement was response compatible (e.g., opening when the correct response was to open) than when it was incompatible (e.g., closing when the correct response was to open) (Stürmer, Aschersleben, \& Prinz, 2000). Although these studies suggest that common mechanisms mediate responding to body movements and to other stimulus classes, they do not test the AIM hypothesis explicitly. Participants in these studies were told to execute a certain response (e.g., to lift a finger) at the onset of a certain stimulus (e.g., finger movement), but they were not instructed to imitate; that is, the relation between the stimulus and response movements (e.g., matching or nonmatching) was not task relevant. The AIM hypothesis proposes that a special-purpose mechanism mediates "active" or intentional imitation, and the present study was designed to test this hypothesis using a task in which participants are instructed to imitate.

In Experiment 1, participants were instructed to imitate lateralized body movements using the same side of their bodies as did the model (e.g., the correct response to a movement of the model's right arm was a matching movement of the participant's right arm). Six different body movements were each presented at six viewing angles. At three of these angles $\left(0^{\circ}, 60^{\circ}\right.$, and $\left.300^{\circ}\right)$, the model had her back turned to the participant, and therefore movements of the right side of her body appeared in the participant's right visual field and required a right response, whereas movements of the left side of the model's body appeared in the participant's left visual field and required a left response. Thus, at angles $0^{\circ}, 60^{\circ}$, and $300^{\circ}$, the task-irrelevant stimulus dimension (location of movement relative to the observer) and the correct response were compatible. At the other three viewing angles $\left(120^{\circ}, 180^{\circ}\right.$, and $\left.240^{\circ}\right)$, the model was facing the participant, and therefore movements of her right side, requiring a right response, appeared in the participant's left visual field, whereas movements of the model's left side, requiring a left response, appeared in the participant's right visual field. Thus, at angles $120^{\circ}, 180^{\circ}$, and $240^{\circ}$, the task-irrelevant stimulus dimension and the response were incompatible.

The AIM hypothesis postulates that the intention to imitate body movements enlists an innate, special-purpose process of actor-centered coding. If, like the products of other special-purpose mechanisms, these actor-centered codes are encapsulated, they should be invulnerable to interference from egocentric codes, and therefore performance on incompatible trials should be as efficient as performance on compatible trials. If, however, the same general mechanisms are involved in S-R translation regardless of a person's intention to imitate, a typical spatial compatibility effect should be observed.

\section{EXPERIMENT 1}

\section{Method}

Participants. Ten participants (19-32 years old) were recruited from within the Department of Psychology, University College London and were paid a small honorarium. All had normal or corrected-to-normal vision and were naive with respect to the purpose of the experiment.

Stimuli and Materials. In a noiseless room with ambient light, participants stood with their toes on a line $150 \mathrm{~cm}$ away from, and parallel to, a screen $(20 \times 21 \mathrm{~cm})$ controlled by a Dell OptiPlex GX1 $\mathrm{PC}$, which was running $3 \mathrm{D}$ Studio software. On each trial, the visual display consisted of a computer graphic representation of a human female, $5.7^{\circ} \times 1.5^{\circ}$ at rest, performing one of the six lateralized body movements, at one of the six viewing angles. When each move- 
ment began, the model was standing with her arms by her sides and her head erect. The six movements were: (1) tilt head to left, (2) tilt head to right, (3) move left leg straight back, (4) move right leg straight back, (5) move left arm straight forward, and (6) move right arm straight forward. For each movement, the model stood at $0^{\circ}-$ that is, with her lateral body axis parallel to that of the participant and her back turned, or, in clockwise rotation, at $60^{\circ}, 120^{\circ}, 180^{\circ}$ (parallel to and facing the participant), $240^{\circ}$ and $300^{\circ}$. Responses, which consisted of imitative body movements, were videotaped for later analysis with a Panasonic NVS1B camcorder and an LG C1-14B monitor with centisecond timer display.

Procedure. Seventy-two trials were presented in two blocks of 36 trials each (six movements viewed at each of six viewing angles). Trial order was randomized, with two constraints: the same movement was not presented more than twice in succession, and viewing angle did not increase or decrease by only one level on more than two successive trials. The duration of each model movement was 2$\mathrm{sec}$, and the interstimulus interval was 3 -sec. The computer generated an auditory stimulus, a beep, as the model reached the full extension of each movement, and this was the participant's signal to respond. The go signal occurred 1-sec after model movement onset, and therefore participants had the opportunity to prepare their response movements in advance. Between responses, participants stood with both feet on the floor, arms straight down at their sides, and heads erect.

The participants were instructed to respond as fast as possible, and to imitate movements of the left side of the model's body with movements of the left side of their own bodies, and vice versa for movements of the right side of the model's body.

An assistant who was unaware of the background and purpose of the experiment scored the videotapes of the participants' perfor- mance. Reaction times (RTs) were calculated manually, using the videotape timer display, by subtracting the time displayed at the auditory go signal from the time displayed at movement onset. Given the naturalistic character of the task, measuring RT with greater precision would have presented significant technical problems. Therefore, the participants were put under time pressure with the intention of using lateral error rate as the primary measure of performance. Participants were scored as having made a lateral error when they produced the same configural body movement as did the model but used the incorrect side of their bodies.

\section{Results and Discussion}

There was a spatial compatibility effect on lateral error (see Figure 1). Mean percentage lateral error was subjected to analysis of variance (ANOVA) with stimulus (left or right) and correct response (left or right) as withinsubjects factors. The main effects of stimulus $(F<1)$ and response $[F(1,9)=1.55]$ were not reliable, but their interaction was significant $[F(1,9)=7.64, p=.02]$. On average, participants made $4.2 \%$ more lateral errors on incompatible than on compatible trials. ${ }^{2}$

The same analysis applied to RTs on correct trials (see Table 1) also indicated a substantial compatibility effect. There was a main effect of stimulus $[F(1,9)=5.45, p=$ $.04]$, indicating that responses to stimuli presented on the participant's left $(1.07 \mathrm{sec})$ were faster than to those presented on the right $(1.13 \mathrm{sec})$, with no effect of response $(F<1)$ and a significant interaction $[F(1,9)=45.82, p<$

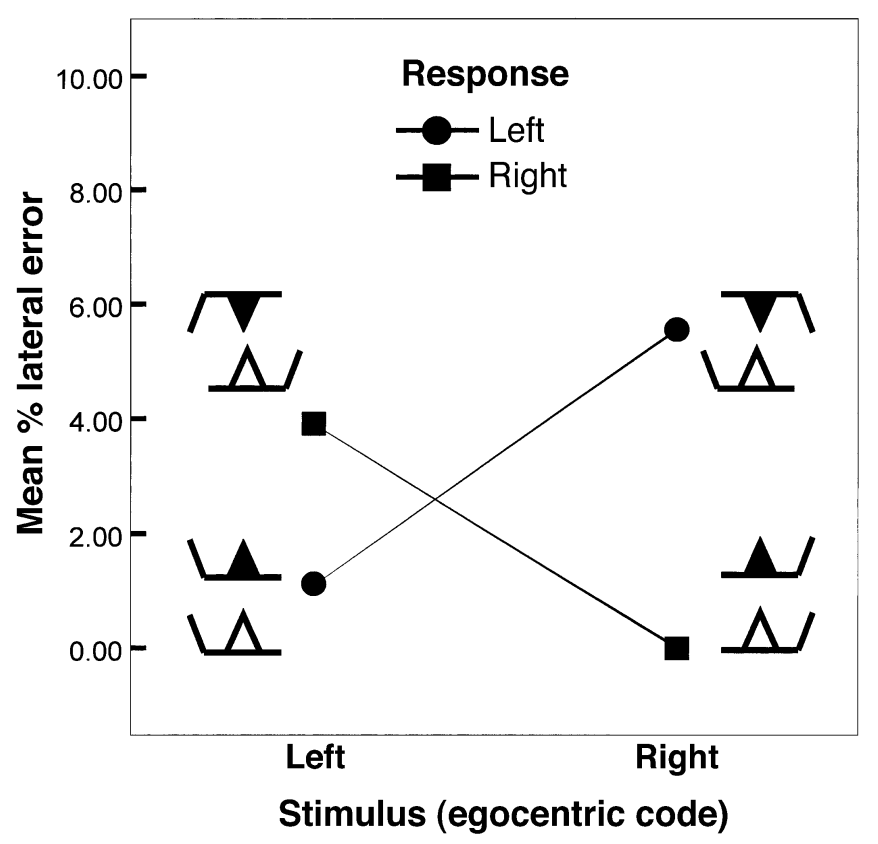

Figure 1. Experiment 1: Congruent $S-R$ mappings. Mean percentage lateral error for body movement stimuli presented to the left and right of the participant's visual field, when task instructions required imitative movement of the left or right of the participant's body. The pair of symbols beside each data point represent the relative positions of the model and the participant in each condition. The open triangle symbol represents the participant making a correct response. The closed triangle symbol represents the model's movement and indicates whether the model was facing the participant $\left(120^{\circ}, 180^{\circ}\right.$, and $\left.240^{\circ}\right)$ or whether her back was turned $\left(0^{\circ}, 60^{\circ}\right.$, and $\left.300^{\circ}\right)$. 
$.0001]$. On average, responses were $.19 \mathrm{sec}$ slower on incompatible than on compatible trials.

In summary, participants required to imitate movements with the same (actor-centered) side of their bodies as the model's were slower to respond, and more likely to use the wrong side of their bodies, when the egocentric location of the stimulus and the correct response were incompatible than when these dimensions were compatible. The results imply that actor-centered coding of body movements for intentional imitation is vulnerable to interference from egocentric codes and therefore provide no support for the view that imitation is mediated by a special process. The results are instead consistent with the view that imitation is mediated by the same general processes that mediate responding to inanimate stimuli with arbitrary S-R mappings.

Dual-route models explain spatial compatibility effects by assuming that stimulus dimensions and responses are coded in a binary fashion, as either "left" or "right" (DeJong, Liang, \& Lauber, 1994). When the stimulus and response codes correspond, S-R translation is facilitated, resulting in faster and/or more accurate responding. However, when the stimulus and response codes do not correspond, there is response conflict because the incorrect response code is activated, making performance slower and/ or more prone to error. Thus, according to a dual-route model, a spatial compatibility effect was observed in Experiment 1 because in compatible trials both the egocentric and the actor-centered spatial codes for the stimulus movement were the same as the response code, but in incompatible trials the egocentric code did not correspond with the response code, therefore generating response conflict.

There is, however, an alternative explanation for the spatial compatibility effect observed in Experiment 1. On trials in which the model was facing $\left(120^{\circ}, 180^{\circ}\right.$, and $\left.240^{\circ}\right)$ the participants, they may have rotated a mental image of the model (or of their own bodies) to make the egocentric stimulus code correspond with the response code. The costs of the mental rotation process could have contributed to the relatively high error rate and slow responding on these trials (Pylyshyn, 1981).

\section{EXPERIMENT 2}

The purpose of Experiment 2 was to investigate whether the spatial compatibility effect observed in Experiment 1 was due to binary coding alone, or to binary coding plus rotation to bring the egocentric stimulus code and the response code into correspondence. The participants in Experiment 2 were presented with the same stimuli as those in Experiment 1, but were now instructed to imitate with the opposite side of their bodies - for example, to move their left arms in response to a right-arm movement. Thus, at viewing angles of $120^{\circ}, 180^{\circ}$, and $240^{\circ}$, when the model was facing the participant, the task-irrelevant stimulus dimension was response compatible, and egocentric responding was correct, but at angles of $0^{\circ}, 60^{\circ}, 300^{\circ}$, when the model's back was turned, the task-irrelevant stimulus dimension was response incompatible, and egocentric responding was incorrect.

If participants engaged in rotation to bring the egocentric stimulus code into correspondence with the response code, then rotation should now occur on trials in which the model's back was turned (compatible trials), but not those in which the model was facing the participant (incompatible trials). Therefore, as in Experiment 1, and in spite of the reversal of instructions across experiments, performance should be better on compatible than on incompatible trials.

By contrast, if the spatial compatibility effect observed in Experiment 1 was due solely to binary coding, one would expect a reverse compatibility effect in Experiment 2-superior performance on incompatible trials. This is because responding on the basis of incongruent S-R mappings, such as those implied by the instruction to imitate with the opposite side of one's body, is known to be vulnerable to "logical recoding." For example, when participants responding to red and green lights pressing a left key colored red and a right key colored green are told to respond to the red light with the green key, and the green light with the red key, RTs are longer on compatible than on incompatible trials (Hedge \& Marsh, 1975). Logical recoding occurs when the reversal rule specified in task instructions is applied, not only to the task-relevant stimulus dimension (actor-centered side of the model's body), but also to the task-irrelevant dimension (egocentric side of the model's body) (Lu \& Proctor, 1994).

\section{Method}

Experiment 2 was identical to Experiment 1 in all respects, except that 9 different participants (19-30 years old) were recruited and they were given incongruent S-R mapping instructions. Specifically, the participants were told to imitate movements of the left side of the model's body with movements of the right side of their own bodies, and vice versa for movements of the right side of the model's body.

\section{Results and Discussion}

Analysis of lateral error rates revealed a reversed spatial compatibility effect (see Figure 2). The main effects of stimulus location $(F<1)$ and correct response location $[F(1,8)=1.20]$ were not reliable, but their interaction was significant $[F(1,8)=6.15, p<.04]$. On average, participants made $12 \%$ more lateral errors on compatible than on incompatible trials.

The RT data (see Table 1) showed a similar trend; on average, RTs were $.05 \mathrm{sec}$ slower on compatible trials than on incompatible trials. This indicates that the effect on lateral error rates was not due to a speed-accuracy tradeoff. An ANOVA applied to the RT data did not reveal any significant effects [stimulus $F(1,8)=2.37$, response $F<1$, stimulus $\times$ response $F(1,8)=1.26]$.

To confirm that the outcomes of the two experiments were different, as would be expected on the basis of previous $\mathrm{S}-\mathrm{R}$ compatibility research with two-dimensional, inanimate stimuli, the data from Experiments 1 and 2 were subjected to ANOVA with stimulus, response, and instruction (congruent vs. incongruent) as factors. In the 


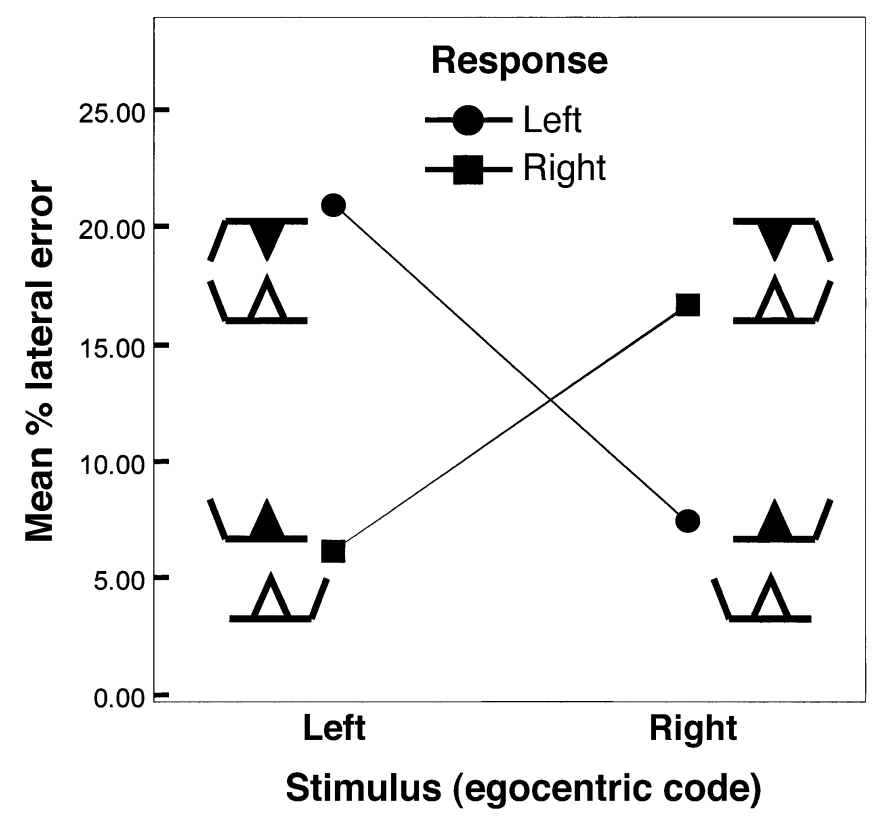

Figure 2. Experiment 2: Incongruent S-R mappings. Mean percentage lateral error for body movement stimuli presented to the left and right of the participant's visual field, when task instructions required imitative movement of the left or right of the participant's body. See Figure 1 for key to symbols.

case of lateral error rates, this revealed a main effect of instruction $[F(1,17)=11.35, p<.004]$, with more errors being made by the incongruent group, and a significant three-way interaction $[F(1,17)=11.12, p<.004]$, confirming that different compatibility effects occurred in Experiments 1 and 2. Similarly, the combined analysis of RTs indicated a stimulus $X$ instruction interaction $[F(1,17)=7.52, p<.02]$ and a reliable three-way interaction $[F(1,17)=20.10, p<.0001]$.

In summary, participants required to imitate the model's movements with the opposite (actor-centered) side of their bodies were slower to respond, and more likely to use the wrong side of their bodies, when the egocentric location of the stimulus and the correct response were compatible, than when these dimensions were incompatible. These re-

Table 1

Mean Reaction Times (in Seconds) as a Function of Stimulus Location (Left or Right of the Participant) and Response Location (Left or Right of the Participant's Body) in Experiments 1 and 2

\begin{tabular}{crc}
\hline & \multicolumn{2}{c}{$\begin{array}{c}\text { Stimulus Location } \\
\text { (Egocentric) }\end{array}$} \\
\cline { 2 - 3 } Response & Left & Right \\
\hline Left & Experiment 1 & \\
Right & 0.98 & 1.23 \\
& 1.16 & 1.03 \\
Left & Experiment 2 & 1.00 \\
Right & 1.08 & 1.08 \\
\hline
\end{tabular}

sults support the view that imitation is mediated by the same binary coding processes that mediate responding in nonimitative spatial compatibility tasks, and rule out the hypothesis that rotation is used to align egocentric stimulus and response codes.

\section{GENERAL DISCUSSION}

The AIM hypothesis suggests that intentional imitation is mediated by a highly efficient, special-purpose mechanism that encodes body movements in an actor-centered fashion. Assuming that a special-purpose mechanism would be encapsulated, and using methods derived from S-R compatibility research, in the present study we found no evidence to support this hypothesis. In both experiments, participants were instructed to imitate actor-centered spatial properties of body movements, yet their performance was affected by task-irrelevant, egocentric spatial properties of the stimuli. In Experiment 1, the participants imitated using the same side of their bodies as the model's, and performance was worse when the location of the stimulus within the observer's visual field was response incompatible than when it was response compatible. In Experiment 2, participants imitated with the opposite side of their bodies, and performance was worse on responsecompatible than on response-incompatible trials.

In order to test the AIM hypothesis, in the present study we assumed that a special-purpose imitation mechanism would be encapsulated. No evidence of encapsulation was found, but this does not rule out the possibility that the 
mechanisms mediating intentional imitation represent distinctive content (organ relations), or that they represent this content in a distinctive code. The latter possibility is more interesting because, at some level of analysis, all representations have distinctive content and because previous work has failed to identify a plausible, nonlinguistic code equating "the felt but unseen movements of the self with the seen but unfelt movements of the other" (Meltzoff \& Decety, 2003). If the existence of such a code is the AIM model's central claim, the model is unlikely to be testable until it specifies the nature of the code.

Like previous work reporting S-R compatibility effects when participants are responding to body movement stimuli without explicit instruction to imitate (e.g., Brass et al., 2001; Stürmer et al., 2000), our results are entirely consistent with the hypothesis that common processes are responsible for imitation and for responding to inanimate stimuli on the basis of arbitrary S-R mappings (Heyes, 2001; Prinz, 2002).

The results of Experiment 2 showed that participants did not use rotation to make the egocentric stimulus code correspond with the response code. However, the results do not completely rule out the possibility that rotation was involved in generating the spatial compatibility effects observed in our experiments. One could argue that participants always engage in rotation when the model is facing them, regardless of whether this produces correspondence (Experiment 1) or noncorrespondence (Experiment 2) between egocentric stimulus and response codes. Mental rotation may be used to achieve correspondence between the egocentric and actor-centered stimulus codes. This alternative rotation hypothesis implies that, in trials in which the model faced the participants in Experiment 2, a rotation strategy was used in preference to mirror responding - that is, to making correct, egocentrically compatible responses.

Whether the results of the present study are due to binary coding alone, or to binary coding plus rotation to align egocentric and actor-centered stimulus codes, they suggest that, in this intentional imitation task, egocentric spatial processing dominated actor-centered spatial processing of body movements. Under the purely binary interpretation, they show that egocentric codes interfered with processing of actor-centered codes. Under the alternative account, they imply that because response selection is preferentially based on egocentric stimulus properties, participants use rotation to convert an actor-centered task into one that affords an egocentric solution.

\section{REFERENCES}

Brass, M., BeKkering, H., \& Prinz, W. (2001). Movement observation affects movement execution in a simple response task. Acta Psychologica, 106, 3-22.

DAUTENHAHN, K., \& Nehaniv, C. L (EDS.) (2002). Imitation in animals and artifacts. Cambridge, MA: MIT Press.

DeJong, R., Liang, C.-C., \& Lauber, E. (1994). Conditional and unconditional automaticity: A dual-process model of effects of spatial stimulus-response correspondence. Journal of Experimental Psychology: Human Perception \& Performance, 20, 731-750.

FoDOR, J. A. (1983). The modularity of mind. Cambridge, MA: MIT Press. Grossman, E. D., \& BlaKe, R. (2002). Brain areas active during visual perception of biological motion. Neuron, 35, 1167-1175.

Hedge, A., \& Marsh, N. W. A. (1975). The effect of irrelevant spatial correspondences on two-choice response-time. Acta Psychologica, 39, 427-439.

HEYES, C. M. (2001). Causes and consequences of imitation. Trends in Cognitive Sciences, 5, 253-261.

Lu, C.-H., \& Proctor, R. W. (1994). Processing of an irrelevant location dimension as a function of the relevant stimulus dimension. Journal of Experimental Psychology: Human Perception \& Performance, 20, 286-298.

MELTZOFF, A. N., \& DeCETy, J. (2003). What imitation tells us about social cognition: A rapprochement between developmental psychology and cognitive neuroscience. Philosophical Transactions of the Royal Society of London: Series B, 358, 491-500.

Meltzoff, A. N., \& Moore, M. K. (1997). Explaining facial imitation: A theoretical model. Early Development \& Parenting, 6, 179-192.

PrinZ, W. (2002). Experimental approaches to imitation. In A. N. Meltzoff \& W. Prinz (Eds.), The imitative mind (pp. 143-162). Cambridge: Cambridge University Press.

Pylyshyn, Z. W. (1981). The imagery debate: Analogue media versus tacit knowledge. Psychological Review, 88, 16-45.

SAmbrooK, T. D. (1998). Does visual perspective matter in imitation? Perception, 27, 1461-1473.

StÜRMER, B., AsCHERSLEBEN, G., \& PrinZ, W. (2000). Correspondence effects with manual gestures and postures: A study of imitation. Journal of Experimental Psychology: Human Perception \& Performance, 26, 1746-1759.

WALLACE, R. J. (1971). S-R compatibility and the idea of a response code. Journal of Experimental Psychology, 88, 354-360.

WAPNER, S., \& Cirillo, L. (1968). Imitation of a model's hand movements: Age changes in transposition of left-right relations. Child Development, 39, 887-894.

\section{NOTES}

1. The idea that distinct mechanisms process animate and inanimate stimuli is consistent with electrophysiological and imaging data identifying neurons that respond selectively to human body movements (Grossman \& Blake, 2002).

2. For both error and RT measures in Experiments 1 and 2, effect sizes were greater at extreme viewing angles $\left(0^{\circ}\right.$ and $\left.180^{\circ}\right)$ than at oblique angles $\left(60^{\circ}, 120^{\circ}, 240^{\circ}\right.$, and $\left.300^{\circ}\right)$. However, there were no significant main effects or interactions involving angle when this factor was included in the analyses.

(Manuscript received January 27, 2003; revision accepted for publication September 8, 2003.) 